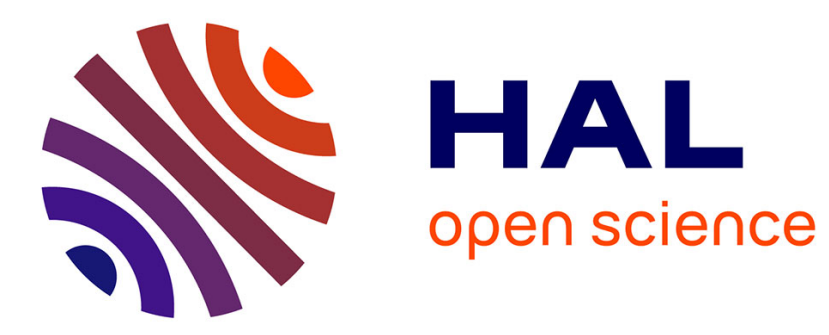

\title{
Perturbing Branching Heuristics in Constraint Solving
}

\author{
Anastasia Paparrizou, Hugues Wattez
}

\section{To cite this version:}

Anastasia Paparrizou, Hugues Wattez. Perturbing Branching Heuristics in Constraint Solving. 26th International Conference on Principles and Practice of Constraint Programming, Sep 2020, Louvainla-Neuve, Belgium. 10.1007/978-3-030-58475-7_29 . hal-03096113

\section{HAL Id: hal-03096113 \\ https://hal.science/hal-03096113}

Submitted on 5 Jan 2021

HAL is a multi-disciplinary open access archive for the deposit and dissemination of scientific research documents, whether they are published or not. The documents may come from teaching and research institutions in France or abroad, or from public or private research centers.
L'archive ouverte pluridisciplinaire HAL, est destinée au dépôt et à la diffusion de documents scientifiques de niveau recherche, publiés ou non, émanant des établissements d'enseignement et de recherche français ou étrangers, des laboratoires publics ou privés. 


\title{
Perturbing Branching Heuristics in Constraint Solving
}

\author{
Anastasia Paparrizou and Hugues Wattez \\ CRIL, University of Artois \& CNRS, France \\ \{paparrizou, wattez\}@cril.fr
}

\begin{abstract}
Variable ordering heuristics are one of the key settings for an efficient constraint solver. During the last two decades, a considerable effort has been spent for designing dynamic heuristics that iteratively change the order of variables as search progresses. At the same time, restart and randomization methods have been devised for alleviating heavy-tailed phenomena that typically arise in backtrack search. Despite restart methods are now well-understood, choosing how and when to randomize a given heuristic remains an open issue in the design of modern solvers. In this paper, we present several conceptually simple perturbation strategies for incorporating random choices in constraint solving with restarts. The amount of perturbation is controlled and learned in a bandit-driven framework under various stationary and non-stationary exploration policies, during successive restarts. Our experimental evaluation shows significant performance improvements for the perturbed heuristics compared to their classic counterpart, establishing the need for incorporating perturbation in modern constraint solvers.
\end{abstract}

\section{Introduction}

For decades now, researchers in Constraint Programming (CP) have put a tremendous effort in designing constraint solvers and advancing their internal components. Many mechanisms have been combined, leading to a technology that is now widely used to solve combinatorial problems. A constraint solver is typically composed of a backtracking search algorithm, a branching heuristic for guiding search, a filtering procedure for pruning the search space, and no-good recording.

Since the very beginning, the order in which variables are selected (assigned) by the branching heuristic holds a central place. It is referred to as the variable ordering heuristic. Choosing the appropriate variable ordering heuristic for solving a given constraint satisfaction problem is quite important since the solving time may vary by orders of magnitude from one heuristic to the other. Recent heuristics are more stable $[15,36]$.

Backtrack search is also vulnerable to unstable behavior because of its inherent combinatorial nature. In the early '00s, the exponential time differences have been investigated under the phase-transition phenomena [19] and the heavytailed distributions of solving time [12]. We can decrease such undesired differences by introducing restart policies, randomization [6] and no-goods recording 
during search. While restarts and no-goods are well established in CP solvers $[11,18,24,27]$, randomization remains limited to ad-hoc techniques that have been found to work well in practice.

In this work, we use randomization to perturb the variable selection process. These perturbations are designed to keep a good and controlled balance between exploitation and exploration. We introduce conceptually simple perturbation strategies for incorporating random choices in constraint solving with restarts. Most of strategies that we present are adaptive, meaning that the amount of perturbation is learned during successive restarts. We exploit the restart mechanism that exists in all modern solvers to control the application of random choices. We deploy a reinforcement learning technique that determines at each run (i.e., at the beginning of the restart), if it will apply the standard heuristic, embedded in the constraint solver, or a procedure that makes random branching choices. This is a sequential decision problem and as such, it can be modeled as a multi-armed bandit problem (MAB) [3], precisely, as a double-armed. In reinforcement learning, the proportion between exploration and exploitation is specified by various policies. We tried several of them, such as Epsilon-greedy [31], EXP3 [4], Thompson Sampling [32], the upper confidence bound UCB1 [4] and MOSS [2]. The learning comes from the feedback taken after each run that reflects the efficiency of the run under a given choice, referred to as a reward function. We also propose a static strategy that perturbs a given heuristic with a fixed probability, found empirically. We evaluate the static and adaptive strategies for several well known heuristics, showing significant performance improvements in favor of the perturbed solver independently of the underlying heuristic used. A perturbed strategy always outperforms its baseline counterpart both in time and number of solved instances. We have also run experiments allowing the use of nogoods, an integral component of solvers nowadays, showing that perturbations still dominate the standard setting, as the no-goods obtained during random runs do not disorientate search.

Many useful observations are derived from this study. The more inefficient a heuristic is, the more effective the perturbation. A perturbed solver can compensate for a potentially bad heuristic choice done by the user, as it permits to automatically improve its performance by visiting unknown parts of the search space. This is due to the random runs, during which the heuristic acquires extra knowledge, other than what obtained when running alone. We show that adaptive strategies always outperform the static ones, as they can adjust their behavior to the instance to be solved and to the heuristic setting. Overall, the results show the benefits of establishing perturbation in $\mathrm{CP}$ solvers for improving their overall performance whatever their default setting is.

\section{Related Work}

Introducing a touch of randomization for better diversifying the search of local and complete procedures has been shown to be quite effective for both SAT (Satisfiability Testing) and CP (Constraint Programming). A stochastic local 
search requires the right setting of the "noise" parameter so as to optimize its performance. This parameter determines the likelihood of escaping from local minima by making non-optimal moves. In GSAT [30], it is referred as the random walk parameter and in walkSAT [29], simply as the "noise". Large Neighborhood Search uses randomization to perform jumps in the search space while freezing a fragment of the best solution obtained so far [26].

In complete CP solvers, the first evidence that diversification can boost search dates back to Harvey and Ginsberg research [17]. Harvey and Ginsberg proposed a deterministic backtracking search algorithm that differs from the heuristic path by a small number of decision points, or "discrepancies". Then, Gomes et al. $[13,12]$ showed that a controlled form of randomization eliminates the phenomenon of "heavy-tailed cost distribution" (i.e., a non-negligible probability that a problem instance requires exponentially more time to be solved than any previously solved instances). Randomization was applied as a tie-breaking step: if several choices are ranked equally, choose among them at random. However, if the heuristic function is powerful, it rarely assigns more than one choice the highest score. Hence, the authors introduced a "heuristic equivalence" parameter in order to expand the choice set for random tie-breaking.

More recently, Grimes and Wallace [14] proposed a way to improve the classical dom/wdeg heuristic (based on constraint weighting) by using random probing, namely a pre-processing sampling procedure. The main idea is to generate the weights of the variables with numerous but very short runs (i.e., restarts) prior search, in order to make better branching decisions at the beginning of the search. Once the weights are initialized, a complete search is performed during which weights either remain frozen or continue updating.

\section{Preliminaries}

A Constraint Network $P$ consists in a finite set of variables vars $(P)$, and a finite set of constraints $\operatorname{ctrs}(P)$. We use $n$ to denote the number of variables. Each variable $x$ takes values from a finite domain, denoted by $\operatorname{dom}(x)$. Each constraint $c$ represents a mathematical relation over a set of variables, called the scope of c. A solution to $P$ is the assignment of a value to each variable in $\operatorname{vars}(P)$ such that all constraints in $\operatorname{ctrs}(P)$ are satisfied. A constraint network is satisfiable if it admits at least one solution, and the corresponding Constraint Satisfaction Problem (CSP) is to determine whether a given constraint network is satisfiable, or not. A classical procedure for solving this NP-complete problem is to perform a backtrack search on the space of partial solutions, and to enforce a property called generalized arc consistency [23] on each decision node, called Maintaining Arc Consistency (MAC) [28]. The MAC procedure selects the next variable to assign according to a variable ordering heuristic, denoted $H$. Then, the selected variable is assigned to a value according to its value ordering heuristic, which is usually the lexicographic order over $\operatorname{dom}(x)$.

As mentioned in Section 2, backtrack search algorithms that rely on deterministic variable ordering heuristics have been shown to exhibit heavy-tailed 
behavior on both random and real-world CSP instances [12]. This issue can be alleviated using randomization and restart strategies, which incorporate some random choices in the search process, and iteratively restart the computation from the beginning, with a different variable ordering [6]. Since our randomization method will be discussed in Section 4, we focus here on restart strategies.

Conceptually, a restart strategy is a mapping res $: \mathbb{N} \rightarrow \mathbb{N}$, where res $(t)$ is the maximal number of "steps" which can be performed by the backtracking search algorithm at run, or trial, $t$. A constraint solver, equipped with the MAC procedure and a restart strategy res, builds a sequence of search trees $\left\langle\mathcal{T}^{(1)}, \mathcal{T}^{(2)}, \ldots\right\rangle$, where $\mathcal{T}^{(t)}$ is the search tree explored by MAC at run $t$. After each run, the solver can memorize some relevant information about the sequence $\left\langle\mathcal{T}^{(1)}, \mathcal{T}^{(2)}, \ldots, \mathcal{T}^{(t-1)}\right\rangle$, like the number of constraint checks in the previous runs, the no-goods that have appeared frequently in the search trees explored so far [20]. The cutoff, $\operatorname{res}(t)$, which is the number of allowed steps, may be defined by the number of nodes, the number of wrong decisions [8], the number of seconds, or any other relevant measure. In a fixed cutoff restart strategy, the number $T$ of trials is fixed in advance, and $\operatorname{res}(t)$ is constant for each trial $t$, excepted for the $T$ th trial which allows an unlimited number of steps (in order to maintain a complete algorithm). This strategy is known to be effective in practice [13], but a good cutoff value res $(t)$ has to be found by trial and error. Alternatively, in a dynamic cutoff restart strategy, the number $T$ of trials is unknown, but res increases geometrically, which guarantees that the whole space of partial solutions is explored after $O(n)$ runs [33]. A commonly used cutoff strategy is driven by the Luby sequence [22].

\section{Perturbation strategies}

As indicated in Section 3, the process of constraint solving with a restart policy may be viewed as a sequence $\langle 1,2, \cdots, T\rangle$ of runs. For the aforementioned restart functions, the sequence of runs is finite, but the horizon $T$ is not necessarily known in advance. During each run $t$, the solver calls the MAC algorithm for building a search tree $\mathcal{T}_{t}$, whose size is determined by the cutoff of the restart policy. If the solver has only access to a single variable ordering heuristic, say $H$, it will run MAC with $H$ after each restart. Yet, if the solver is also allowed to randomize its variable orderings, it is faced with a fundamental choice at each run $t$ : either call MAC with the heuristic $H$ in order to "exploit" previous computations made with this heuristic, or call MAC with a random variable ordering $U$ so as to "explore" new search trees, and potentially better variable orderings. Here, $U$ is any variable ordering drawn at random according to a uniform distribution over the permutation group of $\operatorname{vars}(P)$. We need to highlight here, that the intermediate random runs of $U$ perturb the involved classic heuristic $H$ by updating its parameters, which ultimately affects the behavior/performance of $H$. In other words, the subsequent heuristic runs, will not produce the same orderings as in the traditional solving process, allowing thus the solver to (potentially) tackle instances that neither $H$ nor $U$ would solve stand-alone. 


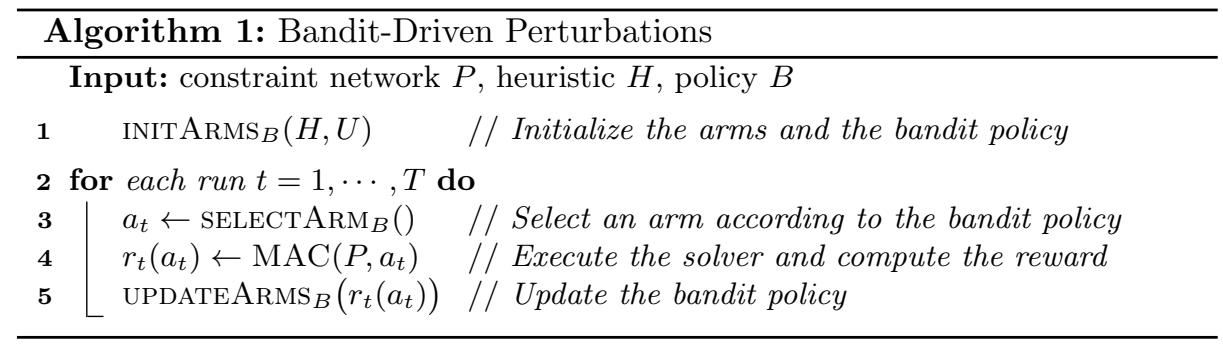

The task of incorporating perturbations into constraint solving with restarts can be viewed as a double-armed bandit problem: during each run $t$, we have to decide whether the MAC algorithm should be called using $H$ (exploitation arm) or $U$ (exploration arm). Once MAC has built a search tree $\mathcal{T}_{t}$, the performance of the chosen arm can be assessed using a reward function defined according to $\mathcal{T}_{t}$. The overall goal is to find a policy mapping each run $t$ to a probability distribution over $\{H, U\}$ so as to maximize cumulative rewards.

Multi-armed bandit algorithms have recently been exploited in CP in different contexts, i.e. for guiding search [21], for learning the right level of propagation [5] or the right variable ordering heuristic $[34,35,37]$. In the framework of Xia and Yap [37], a single search tree is explored (i.e., no restarts), and the bandit algorithm is called at each node of the tree to decide which heuristic to select. The trial is associated with explored subtrees, while in our approach, trials are mapped to runs using a restart mechanism. Our framework makes use of restarts in the same way as the ones of $[34,35]$, as it was shown in [35] that such a framework offers greater improvements compared to the one of [37]. In our case, we utilise a double-armed framework in order to construct our bandit-driven perturbation given by Algorithm 1. The algorithm, takes as input a constraint network $P$, a variable ordering heuristic $H$, and a bandit policy $B$. As indicated above, the bandit policy has access to two arms, $H$ and $U$, where $U$ is the random variable ordering generated on the fly, during execution. The three main procedures used by the bandit policy are INITARMS $B$ for initializing the distribution over $\{H, U\}$ according to policy $B$, SELECTARM $_{B}$ for choosing the arm $a_{t} \in\{H, U\}$ that will be used to guide the search all along the $t$ th run, and UPDATEARMS ${ }_{B}$ for updating the distribution over $\{H, U\}$ according to the observed reward $r_{t}\left(a_{t}\right)$ at the end of the $t$ th run.

\subsection{Reward function}

The feedback $r_{t}\left(a_{t}\right)$ supplied at the end of each run captures the performance of the MAC algorithm, when it is called using $a_{t} \in\{H, U\}$. To this end, the reward function $r_{t}$ maps the search tree $\mathcal{T}_{t}$ built by $\operatorname{MAC}\left(a_{t}\right)$ to a numeric value in $[0,1]$ that reflects the quality of backtracking search when guided by $a_{t}$.

As a reward function, we introduce the measure of the explored sub-tree denoted as esb. esb is given by the number of visited nodes during a run, divided 


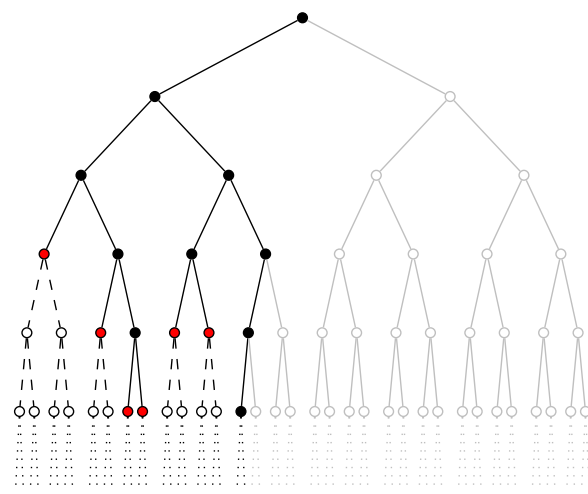

(a) $\operatorname{esb}\left(\mathcal{T}_{\text {left }}\right)=\frac{\log (16)}{\log \left(2^{6}\right)} \approx 0.67$

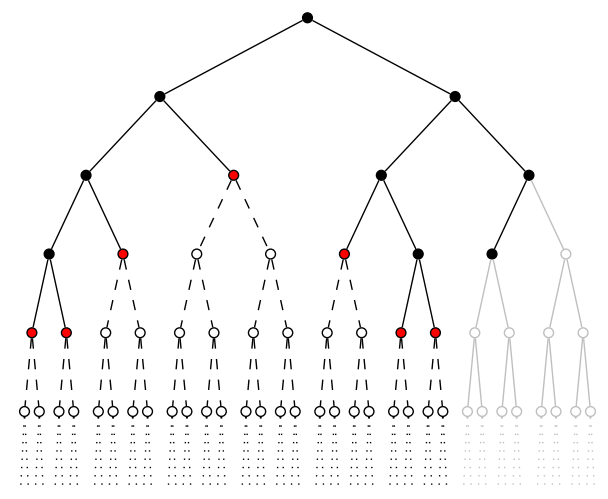

(b) esb $\left(\mathcal{T}_{\text {right }}\right)=\frac{\log (16)}{\log \left(2^{5}\right)} \approx 0.80$

Fig. 1: Comparison of two runs with a restart cutoff fixed to 16 nodes.

by the size of the complete sub-tree defined over the variables selected during this run. The later is simply the size of the Cartesian product of the domains of the variables selected during the run. As selected variables, we consider those ones that have been chosen at least once by the arm in question. esb represents the search space covered by the solver, under a certain setting of a run, compared to the total possible space on the selected variables. The intuition is that an exploration that discovers failures deeply in the tree (meaning that, many variables are instantiated) will be penalized (due to the big denominator) against an exploration that discovers failures at the top branches.

In formal terms, given a search tree $\mathcal{T}$ generated by the MAC algorithm, let $\operatorname{vars}(\mathcal{T})$ be the set of variables that have been selected at least once during exploration of $\mathcal{T}$ and nodes $(\mathcal{T})$ the number of visited nodes. Then,

$$
r_{t}\left(a_{t}\right)=\operatorname{esb}\left(\mathcal{T}_{t}\right)=\frac{\log \left(\operatorname{nodes}\left(\mathcal{T}_{t}\right)\right)}{\log \left(\prod_{x \in \operatorname{vars}\left(\mathcal{T}_{t}\right)}|\operatorname{dom}(x)|\right)}
$$

A logarithmic scaling is needed to obtain a better discrimination between the arms as the numerator is usually significantly smaller than the denominator of the fraction. The reward values belong to $[0,1]$. The higher the ratio/reward is, the better the performance of $a_{t}$ is.

Figure 1, shows a motivating example for the reward function. It displays an example of tree explorations done by two different runs. For simplicity, domains are binary and at each level the variable to be instantiated is fixed per run (namely left and right branches are on the same variable). Empty nodes represent non-visited or pruned nodes, solid black nodes are the visited ones, solid red ones denote failures. Below red nodes, there are the pruned sub-trees in dashed style while the non-visited sub-trees are slightly transparent. For both runs we consider the same number of node visits, i.e. 16. At the left run (Figure 1a), the solver goes until level 6 (selecting 6 variables) while the solver at the right 
run (Figure 1b) goes until level 5. The left run will take the score of 0.67 and the right one 0.80 . Our bandit will prefer the arm that produced the right tree, namely a search that goes faster at the right branches than deeper in the tree (which implies that more search is required). Early failures is a desired effect that many heuristics consider explicitly on implicitly in order to explore smaller search spaces until the solution or unsatisfiability.

\subsection{Perturbation rate}

As indicated in Algorithm 1, the perturbation framework is conceptually simple: based on a restart mechanism, the solver performs each run by first selecting an arm in $\{H, U\}$, next, observing a reward for that arm, and then, updating its bandit policy according to the observed reward. This simple framework, allows us to use a variety of computationally efficient bandit policies to adapt/control the amount of perturbation applied during search. From this perspective, we have opted for five well-studied "adaptive" policies for the double-armed bandit problem, and one "static" (or stationary) policy which serves as reference for our perturbation methods.

$\epsilon$-Greedy. Arguably, this is the simplest adaptive bandit policy that interleaves exploitation and exploration using a parameter $\epsilon$. The policy maintains the empirical means $\hat{r}$ of observed rewards for $H$ and $U$. Initially, both $\hat{r}_{1}(H)$ and $\hat{r}_{1}(U)$ are set to 0 . On each run $t$, the function SELECTARM $\mathrm{AR}_{\epsilon}$ returns with probability $(1-\epsilon)$ the arm $a_{t}$ that maximizes $\hat{r}_{t}\left(a_{t}\right)$, and returns with probability $\epsilon$ any arm $a_{t}$ drawn uniformly at random. Finally, based on the observed reward $r_{t}\left(a_{t}\right)$, the procedure UPDATEARMS ${ }_{\epsilon}$ updates the empirical mean of $a_{t}$ according to

$$
\hat{r}_{t+1}\left(a_{t}\right)=\frac{t}{t+1} \hat{r}_{t}\left(a_{t}\right)+\frac{1}{t+1} r_{t}\left(a_{t}\right)
$$

EXP3. The EXPonentially weighted forecaster for EXPloration and EXPloitation (EXP3) is the baseline bandit policy operating in "non-stochastic" environments, for which no statistical assumption is made about the reward functions [4]. Several variants of EXP3 have been proposed in the literature, but we use here the simplest version defined in [10]. Here, the procedure INITARMS EXP3 sets the initial distribution $\pi_{1}$ of arms to the uniform distribution $(1 / 2,1 / 2)$. During each trial $t$, the procedure SELECTARM EXP3 simply draws an arm $a_{t}$ according to the distribution $\pi_{t}$. Based on the observed reward $r_{t}\left(a_{t}\right)$, the procedure UPDATEARMS ${ }_{\text {EXP3 }}$ updates the distribution $\pi_{t}$ according to the multiplicative weight-update rule:

$$
\pi_{t+1}(a)=\frac{\exp \left(\eta_{t} R_{t}(a)\right)}{\exp \left(\eta_{t} R_{t}(H)\right)+\exp \left(\eta_{t} R_{t}(U)\right)}
$$

$\eta_{t}$ corresponds to the learning rate (usually set to $\frac{1}{\sqrt{t}}$ ),

$$
R_{t}(a)=\sum_{s=1}^{t} \frac{r_{s}(a)}{\pi_{s}(a)} \mathbb{1}_{a \sim \pi_{s}}
$$

and $\mathbb{1}_{a \sim \pi_{s}}$ indicates whether $a$ was the arm picked at trial $s$, or not. 
UCB1. Upper Confidence Bound (UCB) policies are commonly used in "stochastic" environments, where it is assumed that the reward value $r_{t}(a)$ of each arm $a$ is drawn according to a fixed, but unknown, probability distribution. UCB1 is the simplest policy in the Upper Confidence Bound family [3]. In the setting of our framework, this algorithm maintains two 2-dimensional vectors, namely, $n_{t}(a)$ is the number of times the policy has selected arm $a$ on the first $t$ runs, and $\hat{r}_{t}(a)$ is the empirical mean of $r_{t}(a)$ during the $n_{t}(a)$ steps. INITARMS $\mathrm{UCB}_{\mathrm{UC}}$

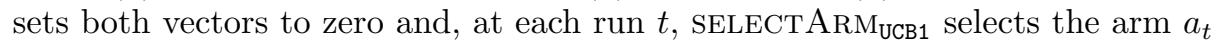
that maximizes

$$
\hat{r}_{t}(a)+\sqrt{\frac{2 \ln (t)}{n_{t}(a)}}
$$

Finally, UPDATEARMS ${ }_{\mathrm{UCB} 1}$ updates the vectors $n_{t}$ and $\hat{r}_{t}$ according to $a_{t}$ and $r_{t}\left(a_{t}\right)$, respectively.

MOSS. The Minimax Optimal Strategy in the Stochastic case (MOSS) algorithm is an instance of the UCB family. The only difference with UCB1 lies in the confidence level which not only takes into account the number of plays of individual arms, but also the number of arms (2) and the number of runs $(t)$. Specifically, SELECT ARM Moss chooses the arm $a_{t}$ that maximizes

$$
\hat{r}_{t}(a)+\sqrt{\frac{4}{n_{t}(a)} \ln ^{+}\left(\frac{t}{2 n_{t}(a)}\right)}
$$

where $\ln ^{+}(x)=\ln \max \{1, x\}$.

TS. The Thompson Sampling algorithm is another well-known policy used in stochastic environments $[1,32]$. In essence, the TS algorithm maintains a beta distribution for the rewards of each arm. INITARMS TS $_{\text {S }}$ sets $\alpha_{1}(a)$ and $\beta_{1}(a)$ to 1 for $a \in\{H, U\}$. On each run $t$, SELECTARM ${ }_{\mathrm{TS}}$ selects the arm $a_{t}$ that maximizes $\operatorname{Beta}\left(\alpha_{t}(a), \beta_{t}(a)\right)$, and UPDATEARMS $\mathrm{Ts}$ uses $r_{t}\left(a_{t}\right)$ to update the beta distribution as follows:

$$
\begin{aligned}
\alpha_{t+1}(a) & =\alpha_{t}(a)+\mathbb{1}_{a=a_{t}} r_{t}\left(a_{t}\right) \\
\beta_{t+1}(a) & =\beta_{t}(a)+\mathbb{1}_{a=a_{t}}\left(1-r_{t}\left(a_{t}\right)\right)
\end{aligned}
$$

$S P$. Finally, in addition to the aforementioned adaptive bandit policies which learn a distribution on $\{H, U\}$ according to observed rewards, we shall consider

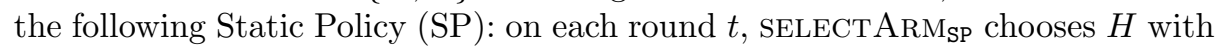
probability $(1-\epsilon)$, and $U$ with probability $\epsilon$. Although this policy shares some similarities with the $\epsilon$-greedy algorithm, there is one important difference: the distribution over $\{H, U\}$ is fixed in advance, and hence, SP does not take into account the empirical means of observed rewards. In other words, UPDATEARMSSP is a dummy procedure that always returns $(1-\epsilon, \epsilon)$. This stationary policy will serve as reference for the adaptive policies in the experiments. 


\section{Experimental Evaluation}

We have conducted experiments on a large dataset to demonstrate the performance of the proposed perturbations. The set includes all instances (612 in total) from the 2017's XCSP3 competition ${ }^{1}$ coming from 60 different problem classes. The experiments have been launched on an $2.66 \mathrm{GHz}$ Intel Xeon and $32 \mathrm{~GB}$ RAM nodes. We have used the $\mathrm{AbsCon}^{2}$ solver in which we integrated our perturbation strategies and the strategies of [13] and [14]. We used 2-way branching, generalized arc consistency as the level of consistency, Luby progression based on node visits as restart policy (the constant is fixed to 100 in AbsCon) and the timeout set to 1,200 seconds. We have chosen a big variety of variable ordering heuristics, including recent, efficient and state-of-the-art ones: dom [16], dom/ddeg [7], activity [25], dom/wdeg [9], CHS [15], wdeg ${ }^{\text {ca.cd }}$ [36] and finally rand which chooses uniformly randomly a variable order. Among these, dom and dom/ddeg do not record/learn anything between two runs (dom and ddeg are re-initialized at the root), while all the others learn during each (random) run and maintain this knowledge all along the solving process, which might change/improve their behavior. We have run all these original heuristics separately for a baseline comparison. Note that in our first experiments no-goods recording are switched off in the solver to avoid biasing the results of heuristics and strategies.

Regarding our perturbation strategies, we denote by SP the static perturbation and by e-greedy, UCB1, MOSS, TS and EXP3 the various adaptive perturbation (AP) strategies. Epsilon of SP and e-greedy are fixed to 0.1. This value has been fixed offline after a linear search of the best value. Apart from comparing to the default solver settings (i.e., original heuristics), we compare to three other perturbation strategies from the bibliography. The one is the sampling algorithm of [14] that corresponds to the sampling pre-processing step which is fixed to 40 restarts with a cutoff of $n$ nodes corresponding to the number of variables of each instance. When the probing phase finishes, we continue updating the variable scoring as it produces better results. The second is the equiv-30 that corresponds to the criterion of equivalence of [13]. This equivalence parameter is set to $30 \%$ as authors proposed. Last, we compare to the standard tie-breaking denoted equiv-0, where a random choice is done among the top ranked variables scored equally by the underlying heuristic. Note that there are no ties, equiv-0 has no effect on the heuristic, as opposed to equiv-30.

Table 1 displays the results of the aforementioned settings and strategies on the XCSP'17 competition dataset. The comparison is given on the number of solved instances (\#inst), within 1, 200 seconds, the cumulative CPU time (time) computed from instances solved by at least one method and the percentage of perturbation (\%perturbation) which is the mean perturbation of the solved instances, computed by the number of runs with the arm $U$ divided by the total of runs. Each time a setting has not solved an instance that another setting solved, it is penalized by the timeout time. Numbers in bold indicate that a strategy

\footnotetext{
${ }^{1}$ See http://www.cril.univ-artois.fr/XCSP17

${ }^{2}$ See http://www.cril.fr/ lecoutre/\#/softwares
} 
Table 1: Comparison of original, sampling, equiv-30 and the proposed perturbed strategies for the XCSP'17 dataset.

\begin{tabular}{|c|c|c|c|c|c|c|c|c|c|}
\hline original & sampling & equiv-0 & equiv-30 & SP & e-greedy & UCB1 & $\begin{array}{l}\text { AP } \\
\text { MOSS }\end{array}$ & TS & EXP3 \\
\hline \#inst 287 & 321 & 312 & 308 & 315 & 314 & $\underline{323}$ & 322 & 318 & $\underline{323}$ \\
\hline dom time (359) 101,589 & 58,496 & 74,064 & 75,992 & 72,460 & 75,474 & $\overline{59,527}$ & 61,171 & 63,856 & $\overline{61,842}$ \\
\hline \%perturb. 0 & - & - & - & 10 & 7.7 & 32.8 & 21.0 & 24.9 & 44.1 \\
\hline \#inst 307 & 321 & 319 & 324 & 343 & 337 & 345 & $\underline{346}$ & 342 & 343 \\
\hline dom/ddeg time (365) 85, 131 & 61,071 & 67,537 & 66,005 & 46,179 & 51,371 & 41,404 & 43,280 & 44,981 & 40,260 \\
\hline \%perturb. 0 & - & - & - & 10 & 8.1 & 34.3 & 21.4 & 26.5 & 45.3 \\
\hline \#inst 342 & 311 & 334 & 329 & $\underline{356}$ & $\underline{356}$ & 352 & 353 & 350 & 351 \\
\hline activity time (372) 52,989 & 82,041 & 60,304 & 64,619 & $\overline{36,688}$ & $\overline{36,125}$ & 39,552 & 37,463 & 40,306 & 39,371 \\
\hline \%perturb. 0 & - & - & - & 10 & 7.6 & 33.5 & 20.7 & 26.0 & 44.4 \\
\hline \#inst 347 & 342 & 349 & 349 & 358 & 346 & 358 & 354 & 356 & $\underline{363}$ \\
\hline dom/wdeg time (381) 55, 599 & 56,038 & 54,276 & 53,263 & 45,615 & 58,888 & 43,726 & 49,052 & 46,896 & $\overline{39,277}$ \\
\hline \%perturb. 0 & - & - & - & 10 & 11.6 & 34.3 & 23.9 & 28.6 & 45.2 \\
\hline \#inst 366 & 354 & 368 & 361 & 370 & 368 & 371 & $\underline{372}$ & 367 & 369 \\
\hline wdeg $^{\text {ca.cd }}$ time (389) 42,565 & 52,344 & 43,944 & 49,717 & 38,966 & 39,292 & 41,745 & 40,433 & 44,941 & 42,661 \\
\hline \%perturb. 0 & - & - & - & 10 & 7.8 & 32.1 & 19.9 & 24.8 & 42.9 \\
\hline \#inst 370 & 343 & 371 & 361 & 371 & 372 & 367 & $\underline{373}$ & 367 & 367 \\
\hline CHS time (389) 41,462 & 64,779 & 42,025 & 56,639 & 37,699 & 38,158 & 43,869 & 38,190 & 46,597 & 42,444 \\
\hline \%perturb. 0 & - & - & - & 10 & 7.3 & 32.6 & 19.9 & 25.7 & 44.5 \\
\hline 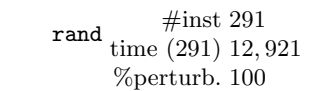 & & & & & & & & & \\
\hline
\end{tabular}

outperformed her corresponding default setting of the solver (i.e., original). Underlined numbers show the winning strategy. dom and dom/ddeg, the unaffected heuristics, appear at the top of the table as they cannot be perturbed by randomized runs. After each run their parameters are reinitialized and not accumulated as for the rest of the heuristics (perturbed ones). Hence, for dom and dom/ddeg, any additional instance that perturbation strategies are able to solve comes from an intervening run of $U$.

The existing perturbation techniques, sampling, equiv-30 and equiv-0, solve more instances than their respective baseline heuristic for the case of dom and dom/ddeg. However, this never happens for sampling and equiv-30 on the more sophisticated heuristics (except from dom/wdeg), where we see that the perturbation they apply disorientates totally the search, solving constantly less instances than the original heuristics (e.g., sampling missed 31 instances for activity). equiv-0 just marginally outperforms pure heuristics by one or two instances while it is far inferior to APs (e.g., it missed 22 instances compared to e-greedy on activity). equiv-30 is superior to sampling on the perturbed heuristics but still far inferior to all proposed strategies. Among the proposed perturbation strategies, we observe that both SP and AP strategies constantly outperform the default setting of the solver, while for many heuristics they have close performance (e.g., activity). MOSS is the best strategy in terms of solved instances and time results, except for activity and dom/wdeg heuristics, where 
SP (e-greedy too) and EXP3 dominate respectively. UCB1 and MOSS are the best strategies for dom and dom/ddeg, with only one instance of difference, showing that a perturbation rate between $20 \%$ and $30 \%$ is the best choice. On the other side, TS with a similar rate seems not to select that well the right arm for all runs. Similarly for equiv-30, which also applies a randomization of $30 \%$ on the top ranked variables, it seems that the way it is applied (i.e., at every decision) is not that efficient. SP and e-greedy, despite winning their original counterparts are less competitive due to their low perturbation rate. EXP3 is also a good candidate policy for dom and dom/ddeg.

rand solved the less instances in total, i.e. 291, in 12,921 seconds, which means that many of them correspond to quite easy instances. As rand is the "bad" arm, a small participation of $10 \%$ in SP is just enough to be beneficial for the heuristics that are by themselves efficient (e.g., wdeg ${ }^{\text {ca.cd }}$, CHS), but as SP is not adaptive, it is rarely better than the AP strategies; it cannot adjust its behavior to heuristics that require more perturbation to improve (e.g., dom). AP strategies, being adaptive, allow usually much more exploration of the $U$ arm that makes them win several instances. An exception is the CHS heuristic, where a perturbation over $20 \%$ might be harmful (i.e., in UCB1, TS and EXP3 policies). Each bandit policy follows a general trend that can vary between heuristics (e.g., UCB1 is around 30\%, MOSS around 20\% and e-greedy with EPX3 represent the two extremities). EXP3 sets the initial distribution $\pi_{1}$ of arms to $(1 / 2,1 / 2)$ such that both arms have equal chances at the beginning. As it is a non-stochastic bandit it needs more exploration than stochastic ones need to converge. Although one would expect that this could deteriorate the solver, it seems that the bandit utilizes the right arm at each run since it is efficient both in time and solved instances. The high perturbation rates come from the mean, that smoothens the high variance between instances. Also, many of the instances are solved fast and EXP3 favorizes a lot $U$, being the best arm at the early (short) runs, while the long runs at the end are done by $H$, which explains its good overall performance. TS despite it is better than original heuristics and existing perturbation methods, it is usually inferior to SP by small differences.

We distinguish the MOSS policy, which apart from being the best policy for many heuristics, it never deteriorates the solver for any heuristic (as happens for some strategies on CHS). It applies the exploration when and where needed (more at the early runs) and converges faster to the best heuristic. Regarding the time performance, all perturbation strategies are faster than their baseline heuristic, even for the most efficient heuristics (i.e., CHS and wdeg ${ }^{\text {ca.cd }}$ ).

Figure 2, visualizes in a cactus plot the performance of the best AP strategy, namely MOSS, for all heuristics compared to their corresponding original heuristic. On x-axis we see the instances solved as time progresses. $\mathrm{y}$-axis displays the allowed time given to the solver. Dashed lines display the performance of original heuristics and solid lines the perturbed solver. The closer a line is to the right bottom corner the more instances has solved in less time. In general, MOSS policy perturbations appear always at the right side of their respective default heuristic. We observe that for the less efficient heuristics, as dom 
and dom/ddeg, the performance gap between the original and the respective perturbed version is big even for easy instance and increases significantly as time passes, corresponding to more difficult instances. It worths noticing that, dom/ddeg after being perturbed, becomes better than activity (solved 4 more instances), that originally was much more efficient than dom/ddeg. dom $_{\text {Moss }}$ solved in total 35 (resp. 39) more instances than dom (resp. dom/ddeg). For activity and dom/wdeg, the curves are closer, though the gap is still significant especially for harder instances. Even for the most efficient heuristics proposed the last two years, namely CHS and wdeg ${ }^{\text {ca.cd }}$, MOSS is almost all the time the best setting for the solver.

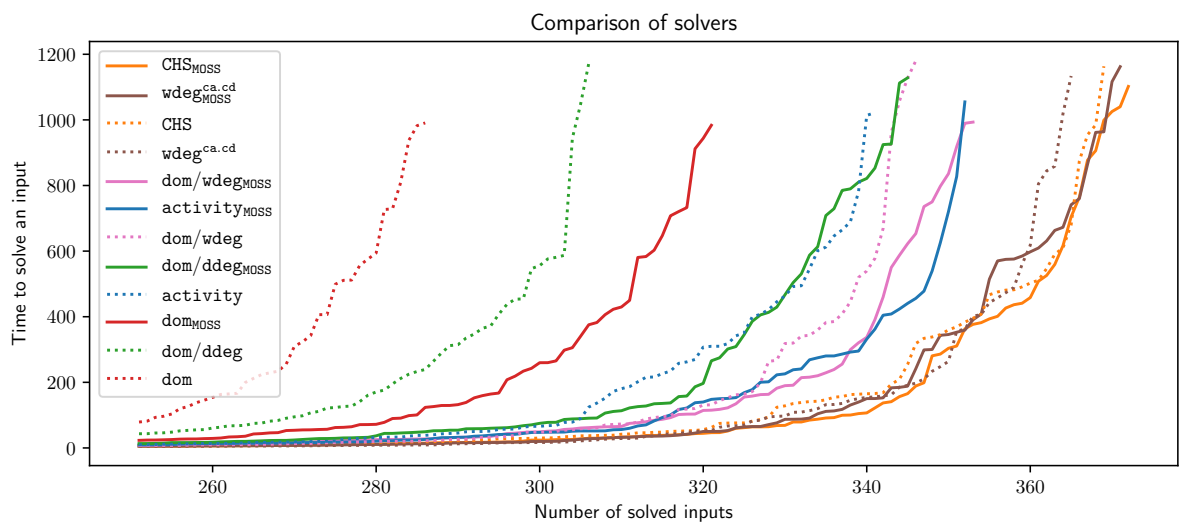

Fig. 2: Comparison of original and MOSS strategies while increasing the allowed time.

In the following, we do not present results for all adaptive strategies, but only for the most stable and efficient ones. We omit presenting results for EXP3 because it failed for CHS, which is one of the two best heuristics in CP, and for TS, because it is never the winning strategy.

Table 2 gives complementary information derived from Table 1 for the best proposed strategies. We have calculated the number of instances solved exclusively by rand (i.e., instances that the heuristic alone could not solve), denoted by \#random, and the number of instances, \#perturb, that the solver solved due to the perturbation rand caused to the corresponding heuristic. Instances in \#perturb are those that are solved neither by the original heuristic nor the rand heuristic, making thus the perturbed solver outperform even its corresponding virtual best solver. As expected, dom and dom/ddeg do not win instances due to perturbation, but only due to a good ordering during a random run (more than 30 instances for each policy). For other heuristics, we see a more balanced distribution of instances in \#random and \#perturb. We observe that the most robust heuristics (CHS and wdeg ${ }^{\text {ca.cd }}$ ) solve extra instances mainly by perturbation (for 
Table 2: Won instances by perturbation or random runs for SP, e-greedy, UCB1 and MOSS with nodes as cutoff for the XCSP'17 dataset

\begin{tabular}{rcccccc}
\hline \multicolumn{7}{c}{ dom dom/ddeg activity dom/wdeg wdeg } \\
\hline Sa.cd & CHS \\
\hline SP \#perturb. & 0 & 0 & 8 & 8 & 5 & 4 \\
\#random & 28 & 36 & 12 & 10 & 3 & 3 \\
e-greedy \#perturb. & 0 & 0 & 8 & 5 & 5 & 6 \\
\#random & 29 & 31 & 10 & 6 & 3 & 2 \\
UCB1 \#perturb. & 0 & 0 & 5 & 7 & 8 & 7 \\
\#random & 38 & 41 & 13 & 10 & 3 & 2 \\
MOSS \#perturb. & 0 & 0 & 6 & 7 & 7 & 10 \\
\#random & 37 & 41 & 12 & 8 & 3 & 3 \\
\hline
\end{tabular}

MOSS: 10 and 7 respectively) rather than by calling rand (3 and 3). Indeed, both are so efficient that the majority of solved instances by rand are also solved by them, which explains why their perturbations 'gain' fewer instances than the other heuristics do. In contrast, activity and dom/wdeg, that are less efficient than CHS and wdeg ${ }^{\text {ca.cd }}$, gain more instances in total, most of which are gained by rand.

Table 3: Comparison of original, SP, e-greedy and MOSS strategies on a subset of families from the XCSP'17 dataset.

\begin{tabular}{|c|c|c|c|c|}
\hline & original & SP & e-greedy & MOSS \\
\hline & dom/ddeg $2(4,803 \mathrm{~s}, 0 \%)$ & $3(4,533 \mathrm{~s}, 10 \%)$ & $2(4,803 \mathrm{~s}, 3 \%)$ & $4(2,952 \mathrm{~s}, 17 \%)$ \\
\hline CoveringArray & dom/wdeg $4(2,669 \mathrm{~s}, 0 \%)$ & $4(3,450 \mathrm{~s}, 10 \%)$ & $3(3,605 \mathrm{~s}, 31 \%)$ & $5(2,156 \mathrm{~s}, 34 \%)$ \\
\hline & CHS $4(2,483 \mathrm{~s}, 0 \%)$ & $4(2,424 \mathrm{~s}, 10 \%)$ & $5(1,705 s, 3 \%)$ & $\overline{6(1,491 \mathrm{~s}, 17 \%)}$ \\
\hline \multirow{3}{*}{ SuperSolutions } & dom $2(8,404 \mathrm{~s}, 0 \%)$ & $4(6,972 \mathrm{~s}, 10 \%)$ & $6(7,471 \mathrm{~s}, 6 \%)$ & $6(3,912 \mathrm{~s}, 24 \%)$ \\
\hline & wdeg $^{\text {ca.cd }} 5(4,968 \mathrm{~s}, 0 \%)$ & $7(3,101 \mathrm{~s}, 10 \%)$ & $6(4,467 \mathrm{~s}, 9 \%)$ & $7(3,821 \mathrm{~s}, 23 \%)$ \\
\hline & CHS $7(3,507 \mathrm{~s}, 0 \%)$ & $\overline{6(3,788 \mathrm{~s}, 10 \%)}$ & $6(3,812 \mathrm{~s}, 6 \%)$ & $8(1,689 \mathrm{~s}, 20 \%)$ \\
\hline \multirow{3}{*}{ KnightTour } & dom/ddeg $5(7,220 \mathrm{~s}, 0 \%)$ & $9(3,276 \mathrm{~s}, 10 \%)$ & $9(2,623 \mathrm{~s}, 34 \%)$ & $9(3,092 \mathrm{~s}, 37 \%)$ \\
\hline & activity $7(4,891 \mathrm{~s}, 0 \%)$ & $9(3,530 \mathrm{~s}, 10 \%)$ & $\overline{9(2,823 \mathrm{~s}, 26 \%)}$ & $9(2,513 \mathrm{~s}, 30 \%)$ \\
\hline & dom/wdeg $5(7,218 \mathrm{~s}, 0 \%)$ & $9(4,302 \mathrm{~s}, 10 \%)$ & $7(5,509 \mathrm{~s}, 25 \%)$ & $9(3,286 \mathrm{~s}, 39 \%)$ \\
\hline \multirow{3}{*}{ Blackhole } & dom $6(2,609 \mathrm{~s}, 0 \%)$ & $6(2,489 \mathrm{~s}, 10 \%)$ & $6(2,503 \mathrm{~s}, 4 \%)$ & $6(2,423 \mathrm{~s}, 16 \%)$ \\
\hline & dom/ddeg $8(327 \mathrm{~s}, 0 \%)$ & $8(44 \mathrm{~s}, 10 \%)$ & $8(55 \mathrm{~s}, 4 \%)$ & $8(25 \mathrm{~s}, 20 \%)$ \\
\hline & activity $8(897 \mathrm{~s}, 0 \%)$ & $7(1,618 \mathrm{~s}, 10 \%)$ & $8(908 \mathrm{~s}, 6 \%)$ & $8(816 \mathrm{~s}, 17 \%)$ \\
\hline \multirow{3}{*}{ LatinSquare } & dom $8(5,841 \mathrm{~s}, 0 \%)$ & $8(5,840 \mathrm{~s}, 10 \%)$ & $8(5,928 \mathrm{~s}, 2 \%)$ & $8(5,813 \mathrm{~s}, 13 \%)$ \\
\hline & dom/wdeg $10(2,481 \mathrm{~s}, 0 \%)$ & $12(1,143 \mathrm{~s}, 10 \%)$ & $10(3,262 \mathrm{~s}, 44 \%)$ & $\overline{10(3,351 \mathrm{~s}, 40 \%)}$ \\
\hline & CHS $11(3,031 \mathrm{~s}, 0 \%)$ & $9(3,947 \mathrm{~s}, 10 \%)$ & $9(4,469 \mathrm{~s}, 3 \%)$ & $8(5,866 \mathrm{~s}, 13 \%)$ \\
\hline
\end{tabular}

As the results in Table 1 are quite condensed, in Table 3 we show how strategies operate and adjust for certain problem classes. For each class and each heuristic, we present the number of solved instances, the total time and the perturbation rate. For CoveringArray, MOSS is the best strategy to apply the ap- 
Table 4: Comparison of original, sampling, equiv-30, SP, e-greedy and MOSS with nodes as cutoff and no-goods activated.

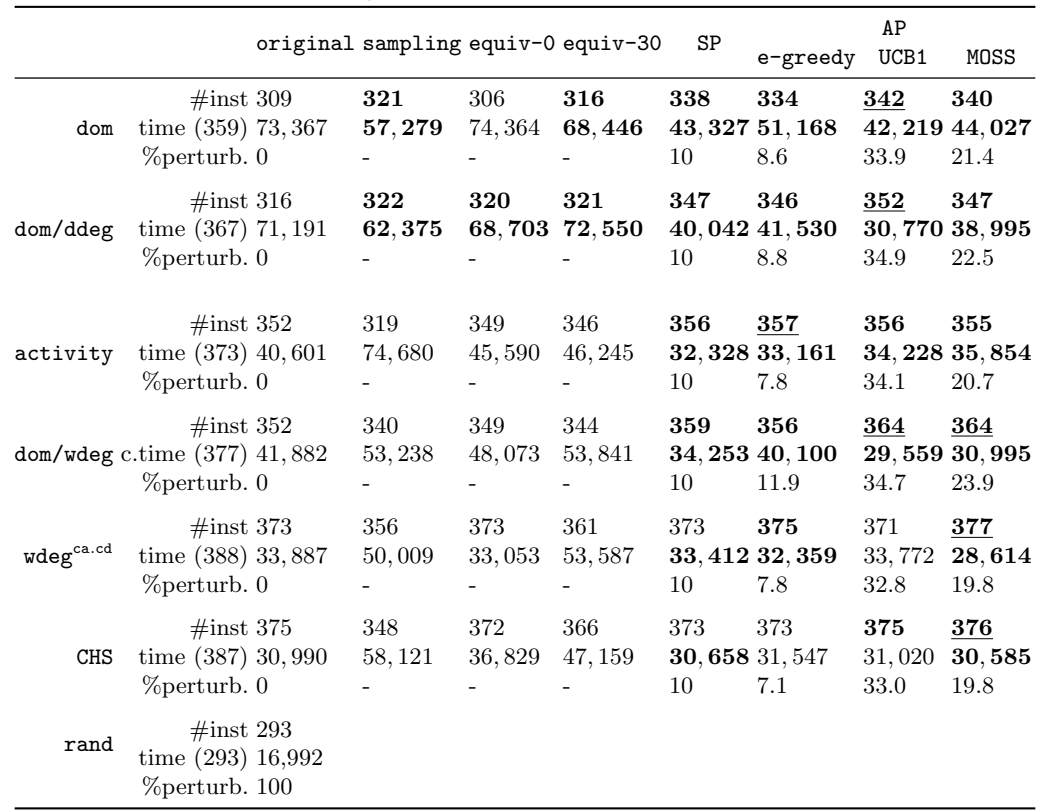

propriate perturbation rate, independently of the heuristic chosen, compared to SP and e-greedy whose rate is too low. For SuperSolutions with dom as heuristic method, we see that e-greedy and MOSS are both winners despite their totally different rates. Though, it is notable that MOSS is twice faster than e-greedy on the same instances. SP with a rate close to e-greedy wins the half instances compared to it (and MOSS). Such observations are clear evidences, that not only the amount of randomization counts but also the when it appears. Policies as MOSS learn to discriminate on which run to apply $H$ or $U$. Recall that, compared to other policies, MOSS considers in SELECTARM MOss $_{\text {more parameters, as }}$ the number or arms and the number of runs. In KnightTour, all strategies are efficient, but APs are always better than SP in terms of time. For Blackhole and LatinSquare, perturbation is not fruitful and thus, both APs converge to very low rates even when instances are easy (just few seconds per instance). Notice that, in general dom/wdeg is helped a lot by perturbation, as in all classes rates are higher compared to other heuristics (double percentages). Also, the percentage of perturbation varies a lot depending on the heuristic and the problem class, which is the reason of the success of APs.

As modern solvers exploit no-goods to improve their overall performance, we repeated our experiments by activating no-goods in order to examine the robustness of the proposed strategies and the interaction between no-goods and perturbation. Table 4 displays the results sampling, equiv-0, equiv-30, SP and 
the best AP strategies, namely e-greedy, UCB1 and MOSS. As seen in Table 1, sampling and equiv-30 make some improvements on the less efficient heuristics as dom and dom/ddeg, but are still inferior to SP and MOSS, while they are inefficient on all other heuristics. Surprisingly, equiv-0, despite being still more efficient than sampling and equiv-30, seems to interact badly with the presence of no-goods, as it can no longer improve the solver for any heuristic (just marginally dom/ddeg). SP, despite being static, it remains efficient apart from the case of CHS. Regarding the AP strategies, e-greedy (resp. UCB1) wins almost always the underlying heuristic except from the case of CHS (resp. wdeg $^{\text {ca.cd }}$ ). MOSS is again the most stable strategy, being able to improve all heuristics it perturbed. Note that the presence of no-goods has improved the performance of both the default and the perturbed solver. Therefore, there are slightly smaller differences between them compared to Table 1. The proposed perturbation strategies are robust to this fundamental parameter for solvers compared to existing strategies and adapt their behavior, especially MOSS.

\section{Conclusion}

We presented several strategies that significantly improve the performance and robustness of the solver by perturbing the default branching heuristic. It is the first time an approach tries to learn how and when to apply randomization in an on-line and parameter-free fashion. Controlled random search runs help variable ordering heuristics to acquire extra knowledge from parts of the search space that they were not dedicated to explore. We summarize the benefits of our approach which are manifold:

- Our perturbation techniques constantly improve the performance of the solver independently of the heuristic used as baseline in the solver. A perturbed strategy always outperforms its baseline counterpart both in time and solved instances.

- The presence of no-goods does not impact the efficacy of the perturbed solver. The produced no-goods are still fruitful.

- Perturbed heuristics can compensate for a wrong heuristic choice done by the user. Thanks to perturbation, the performance of the solver with a bad initial heuristic can reach or even outperform the performance of the solver with a better baseline heuristic. This is a step towards autonomous and adaptive solving, where the solver learns and adjusts its behavior to the instance being solved.

- Our approach is generic and easy to embed in any solver that exploits restarts.

Acknowledgments. The authors would like to thank Frederic Koriche for his valuable advices on the Machine Learning aspects of the paper as well as the anonymous reviewers for their constructive remarks. This work has been partially supported by the project Emergence 2020 BAUTOM of INS2I and the project CPER Data from the region "Hauts-de-France". 


\section{References}

1. Agrawal, S., Goyal, N.: Near-optimal regret bounds for Thompson Sampling. J. ACM 64(5), 30:1-30:24 (2017)

2. Audibert, J.Y., Bubeck, S.: Minimax policies for adversarial and stochastic bandits. In: COLT. pp. 217-226. Montreal, Canada (2009)

3. Auer, P., Cesa-Bianchi, N., Fischer, P.: Finite-time analysis of the multiarmed bandit problem. Machine Learning 47(2), 235-256 (May 2002)

4. Auer, P., Cesa-Bianchi, N., Freund, Y., Schapire, R.: The nonstochastic multiarmed bandit problem. SIAM Journal on Computing 32(1), 48-77 (2002)

5. Balafrej, A., Bessiere, C., Paparrizou, A.: Multi-armed bandits for adaptive constraint propagation. In: Proceedings of IJCAI'15. pp. 290-296 (2015)

6. van Beek, P.: Backtracking search algorithms. In: Handbook of Constraint Programming, chap. 4, pp. 85-134. Elsevier (2006)

7. Bessiere, C., Régin, J.: MAC and combined heuristics: two reasons to forsake FC (and CBJ?) on hard problems. In: Proceedings of CP'96. pp. 61-75 (1996)

8. Bessiere, C., Zanuttini, B., Fernandez, C.: Measuring search trees. In: Proceedings of ECAI'04 workshop on Modelling and Solving Problems with Constraints. pp. 31-40 (2004)

9. Boussemart, F., Hemery, F., Lecoutre, C., Sais, L.: Boosting systematic search by weighting constraints. In: Proceedings of ECAI'04. pp. 146-150 (2004)

10. Bubeck, S., Cesa-Bianchi, N.: Regret Analysis of Stochastic and Nonstochastic Multi-armed Bandit Problems. Foundations and Trends in Machine Learning, Now Publishers (2012)

11. Gecode Team: Gecode: Generic constraint development environment (2006), available from http://www.gecode.org

12. Gomes, C., Selman, B., Crato, N., Kautz, H.: Heavy-tailed phenomena in satisfiability and constraint satisfaction problems. Journal of Automated Reasoning 24(1), 67-100 (2000)

13. Gomes, C.P., Selman, B., Kautz, H.: Boosting combinatorial search through randomization. In: Proceedings of AAAI '98. pp. 431-437 (1998)

14. Grimes, D., Wallace, R.: Sampling strategies and variable selection in weighted degree heuristics. In: Proceedings of CP'07. pp. 831-838 (2007)

15. Habet, D., Terrioux, C.: Conflict History Based Branching Heuristic for CSP Solving. In: Proceedings of the 8th International Workshop on Combinations of Intelligent Methods and Applications (CIMA). Volos, Greece (Nov 2018)

16. Haralick, R., Elliott, G.: Increasing tree search efficiency for constraint satisfaction problems. Artificial Intelligence 14, 263-313 (1980)

17. Harvey, W.D., Ginsberg, M.L.: Limited discrepancy search. In: Proceedings of the 14th International Joint Conference on Artificial Intelligence - Volume 1. p. 607-613. IJCAI'95 (1995)

18. Hebrard, E.: Mistral, a constraint satisfaction library. Proceedings of the Third International CSP Solver Competition 3(3), 31-39

19. Hogg, T., Huberman, B.A., Williams, C.P.: Phase transitions and the search problem. Artificial Intelligence 81(1), 1 - 15 (1996)

20. Lecoutre, C., Sais, L., Tabary, S., Vidal, V.: Recording and minimizing nogoods from restarts. Journal on Satisfiability, Boolean Modeling and Computation (JSAT) 1, 147-167 (2007)

21. Loth, M., Sebag, M., Hamadi, Y., Schoenauer, M.: Bandit-based search for constraint programming. In: Proceedings of CP'13. pp. 464-480 (2013) 
22. Luby, M., Sinclair, A., Zuckerman, D.: Optimal speedup of las vegas algorithms. Information Processing Letters 47(4), 173-180 (1993)

23. Mackworth, A.: On reading sketch maps. In: Proceedings of IJCAI'77. pp. 598-606 (1977)

24. Merchez, S., Lecoutre, C., Boussemart, F.: Abscon: a prototype to solve CSPs with abstraction. In: Proceedings of CP'01. pp. 730-744 (2001)

25. Michel, L., Hentenryck, P.V.: Activity-based search for black-box constraint programming solvers. In: Proceedings of CPAIOR'12. pp. 228-243 (2012)

26. Pisinger, D., Ropke, S.: Large neighborhood search. In: Handbook of metaheuristics. pp. 399-419. Springer (2010)

27. Prud'homme, C., Fages, J.G., Lorca, X.: Choco Solver Documentation. TASC, INRIA Rennes, LINA CNRS UMR 6241, COSLING S.A.S. (2016), http://www. choco-solver.org

28. Sabin, D., Freuder, E.: Contradicting conventional wisdom in constraint satisfaction. In: Proceedings of CP'94. pp. 10-20 (1994)

29. Selman, B., Kautz, H.A., Cohen, B.: Noise strategies for improving local search. In: Proceedings of AAAI '94. pp. 337-343 (1994)

30. Selman, B., Levesque, H., Mitchell, D.: A new method for solving hard satisfiability problems. In: Proceedings of the Tenth National Conference on Artificial Intelligence. pp. 440-446. AAAI'92, AAAI Press (1992), http://dl.acm.org/citation.cfm? id $=1867135.1867203$

31. Sutton, R., G. Barto, A.: Reinforcement learning: An introduction 9, 1054 (02 1998)

32. Thompson, W.R.: On the likelihood that one unknown probability exceeds another in view of the evidence of two samples. Biometrika 25(3-4), 285-294 (12 1933)

33. Walsh, T.: Search in a small world. In: Proceedings of IJCAI'99. pp. 1172-1177 (1999)

34. Wattez, H., Koriche, F., Lecoutre, C., Paparrizou, A., Tabary, S.: Heuristiques de recherche : un bandit pour les gouverner toutes. In: 15es Journées Francophones de Programmation par Contraintes - JFPC 2019 (2019), https://hal. archives-ouvertes.fr/hal-02414288

35. Wattez, H., Koriche, F., Lecoutre, C., Paparrizou, A., Tabary, S.: Learning variable ordering heuristics with multi-armed bandits and restarts. In: Proceedings of ECAI'20 (to appear)

36. Wattez, H., Lecoutre, C., Paparrizou, A., Tabary, S.: Refining constraint weighting. In: Proceedings of ICTAI'19. pp. 71-77 (2019)

37. Xia, W., Yap, R.H.C.: Learning robust search strategies using a bandit-based approach. In: Proceedings of AAAI'18. pp. 6657-6665 (2018) 